muscular fibres and fascia around the gut, and on other con siderations which pass beyond the limits of this paper.

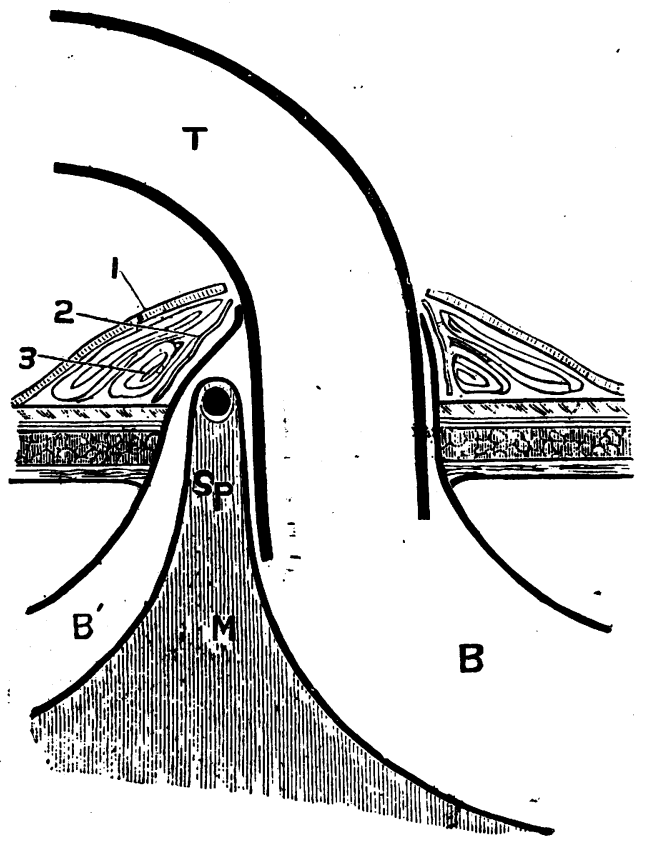

Fig. 3.-Diagram to show method of performing colostomy. B, bowel above spur; $B^{\prime}$, bowel collapsed below spur; T, rubber tubing; sp., spur. At the top of the spur the black circle represents section of a supporting stiff rod.

As regards the age of the patient, special remark is scarcely called for, for it is now well recognised that mere age is no barrier to surgical operation. I have successfully amputated for gangrene the great toe of a gentleman, in his 98th year. I have made a complete excision of the mamma for cancer in a lady, aged 89, who is still alive and well in her 91st year. I have performed at least ten ovariotomies in patients between 70 and 80 . If the patient is well guarded against shock and the operation is quickly performed recovery is quite as satisfactory in the aged as in the young.

\section{A MODIFICATION OF THE OPERATION OF PYLOROPLASTY,}

With a Report of Two Cases.

BY A. W. MAYO ROBSON, F.R.C.S.,

Vice-President of the Surgical Section of the British Medical Association; Senior Surgeon to the General Infirmary at Leeds; Professor of Burgery in the Yorkshire College of the Victoria University.

THE principle of obtaining increased calibre in a strictured channel by dividing the narrow passage longitudinally and suturing the wound transversely, is as ingenious as it is efficient, and in no situation can such a procedure be of more service than in simple stricture of the pylorus, where the operation is known as pyloroplasty.

The invention belongs to Heineke, who performed his first operation in March, 1886, though the name of Mikulicz, who did the second in January, 1887, is sometimes attached to the procedure.

Simple stricture of the pylorus is far from common, and therefore the experience of any one surgeon must be somewhat limited : this must be my excuse for basing my conclusions on so small a number as two cases.

I think there can be no doubt that pyloroplasty will completely replace Loreta's operation, since with no greater or perhaps even less immediate risk, it affords a more certain prospect of freedom from relapse.

1 Bince writing this paper I have had an equally successful third case in which after operation, the patient gained 2 stones in weight within
the month.
The modification of the operation by the use of the bone bobbin which I have adopted, not only expedites the procedure, but in my opinion renders it safer than when simple suture is employed. The following diagrams show better than words the original and the modified operations:

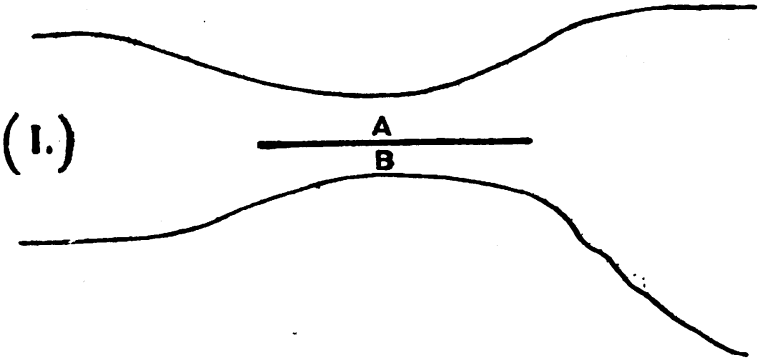

Fig. 1. First stage.

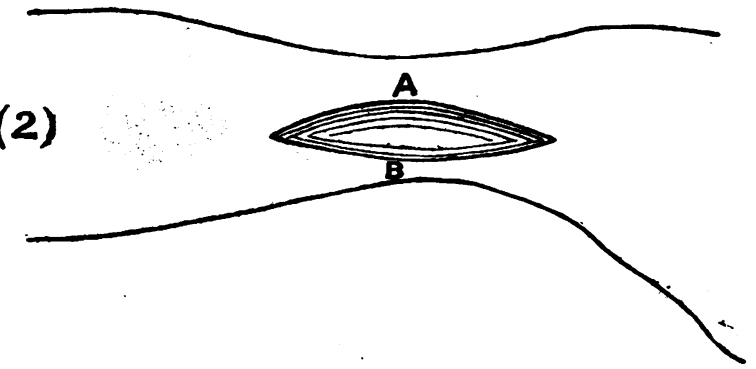

Fig. 2.-Second stage.

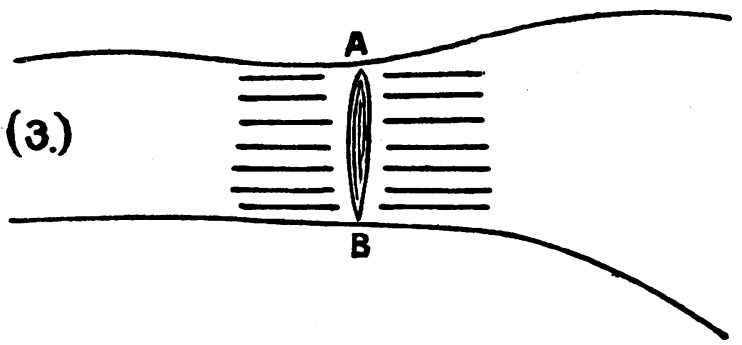

Fig. 3.-Third stage of the original method.

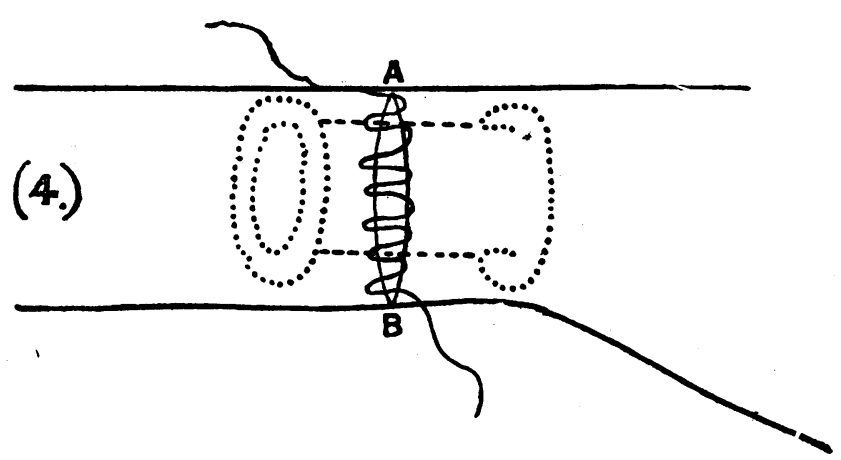

Fig. 4.-Third stage of the modified method as employed in the cases related.

It will be seen that by the use of a decalcified bone bobbin only two continuous sutures are required, the first to unite the mucous margins, the second the serous. The bone tube further secures an immediately and thoroughly patent channel, and affords protection to the line of sutures for from twenty-four to forty-eight hours, when union.should be well established. The account of the following cases proves that the operation is both feasible in execution and satisfactory in results.

CABE I.-R. F., aged 34, residing in Scarborough, was sent to me by his medical attendant, and admitted to the Leeds 
General Infirmary, January 10th, 1895. He gave the history of having been well up to five years before, when he began to have pain after food and vomiting, but he did not remember having vomited blood. His symptoms continued off and on until June, 1894, when he ceased work and sought advice. Between Christmas, 1892, and January, 1895, his weight diminished from 9 st. 5 lbs. to $7 \mathrm{st} .5 \mathrm{lbs}$.

On admission he was much emaciated, and the signs of dilated stomach were well marked. After taking food he had no rest until vomiting occurred; the vomit was sour smelling and yeasty but never contained bile or blood. He suffered much, not only from painful cramps in the extremities, which required morphine for their relief, but also from distressing spasms of the stomach, which could be distinctly felt contracting, the outline of the dilated organ showing through the abdominal walls. At times a sausage-shaped hard swelling could be felt a little above and to the right of the umbilicus; but as this was only occasionally felt, it was manifestly a contracting pylorus and not a permanent tumour. The stomach was washed out daily and the patient fed with peptonised food, but as all food produced pain and was rejected, rectal feeding was adopted. January 24th. Pyloroplasty was performed through an incision 3 inches long in the linea alba above the umbilicus. The pylorus was found to be surrounded by adhesions and tucked up to the under surface of the liver; its outer surface was puckered as if an alcer had approached the peritoneal coat and had then healed without perforation. On opening the stomach close to the pylorus it was found that the pyloric orifice was contracted to the size of a No. 6 catheter, and that it was surrounded by cicatricial tissue. No shock followed the operation, and the after-progress was all that could be desired. Feeding by the mouth was begun on January 26 th and the rectal feeding discontinued on January 29th. After the sickness due to the anæsthetic had passed off there was no further vomiting. The sutures were removed on the seventh day, when the wound was found to be healed. He rapidly gained flesh, and was discharged well and strong on March 2nd, having gained nearly a stone in weight, and being able to take ordinary food in full meals.

CAsE Ir.-W. F., aged 52, sent to me by Dr. Cheeswright, of Rotherham, on account of vomiting and loss of flesh, was admitted to the Leeds General Infirmary in the first week of April, 1895. He gave the history of indigestion for two years, but of being otherwise fairly well up to Christmas, 1894, when he began to have pain about two hours after food, which was at times followed by vomiting. The vomiting recurred every other day, when a large quantity of mucus and partlydigested food would be rejected. The vomit at first contained dark blood, though during the month before admission no blood had been noticed. During the early part of his illness there was marked tenderness over the epigastrium, but before admission the tenderness disappeared. He was not weighed until five weeks before admission, but in that short time he had lost a stone and a-half in weight.

On admission he had a starved look, and was feeble generally. The signs of dilated stomach were well marked, but no distinct tumour could be felt in the region of the pylorus. There were no other signs of disease. The stomach was washed out daily with a solution of boracic acid, and on April 8th pyloroplasty was performed through an incision 3 inches long in the middle line above the umbilicus. On exposure, the pylorus was found to be much thickened and surrounded by adhesions, which fixed it firmly to the under surface of the liver. After detaching adhesions it could be brought down sufficiently far to be manipulated. On making the longitudinal incision through the pylorus the channel was found to be surrounded by cica. tricial material, leaving a passage which would only admit a No. 10 catheter. The operation, with the use of the bone bobbin, was performed as shown in the diagrams. The afterprogress was perfectly satisfactory, and he began to take food by the mouth on the second day. In the second week he was taking solid food freely, and before 'going home at the month end he was eating large meals of the ordinary hospital diet. He never vomited after the operation, and the wound healed by first intention. When he left for home he had gained half a stone in weight and looked well.

With a little manipulative dexterity, the operation pyloroplasty can be done so quickly and with so little exposure of viscera that post-operative shock need hardly be feared, and as the incision through the pylorus is through cicatricial tissue there is little or no fear of hæmorrhage; in fact, in the two cases related, not a single ligature was required. The only difficulty, and it is one which may usually be expected in these cases, is that the pylorus has to separated from adhesions set up by the inflammatory process which has caused the stricture. These are better separated by the fingers than by a cutting instrument.

It will be found to facilitate the application of the sutures if the longitudinal incision through the pylorus is made rather nearer the lower margin, so that when the slit is sutured transversely the upper angle is well within view. As regards sutures, fine catgut is the best for uniting the mucous margins and fine silk for the serous surfaces.

For simple stricture of the intestine $I$ have found the same operation to prove equally satisfactory.

\section{HUNTERIAN LECTURES}

ON THE

\section{EXPERIENCE OF ST. GEORGE'S HOSPITAL IN LAPAROTOMY;}

\section{EXCLUSIVE OF GYN ACOLOGICAL OPERATIONS,} FROM 1888 TO 1894 INCLUSIVE.

Delivered at St. George's Hospital Medrcal School

BY T. HOLMES, F.R.C.S. Consulting Surgeon and Treasurer to the Hospital.

Lecture III.-Gastrojrjunostomy : Internal StrangulaTION AND STRICTURE OF BOWEL. Conclusion.

THE operation of gastrojejunostomy appears to mark another real advance in surgery, attaining without inordinate risk in cases of pyloric obstruction the object which surgeons have long pursued, but with little success, by gastrostomy, in cases of cesophageal obstruction, namely, the prolongation of life in tolerable comfort and with the power of taking natural food. And I believe that this operation has been so successful that it has displaced or will displace the complicated and dangerous procedure of pylorectomy.

Our experience of gastrojejunostomy during the period covered by these notes has consisted of four cases, in three of which complete success was obtained. The fatal case was that of a woman, under Mr. Haward's care, advanced in life (aged 75) and suffering from cancer in the groin as well as in the stomach. She died on the fifth day after the operation. The necropsy furnished this interesting specimen, which shows the small but adequate artificial communication provided by the operation and also the total disappearance of the Senn's bone plates used to approximate the viscera.

In the successful cases one of the most satisfactory features was the rapidity with which the patient recovered appetite, strength, and flesh. Thus in a case under Mr. Bennett's care in 1891, published in Clin. Trans., xxiv., 247, the patient had been suffering from nearly or quite complete obstruction of the pylorus and was in the last stage of emaciation. The operation was performed on February 27 th, and he was dis. charged on April 23rd. During these two months he had increased $2 \frac{1}{2}$ stone in weight. I may notice here that his weakness was so extreme that it was necessary to feed him by the mouth even on the evening of the operation, when egg and brandy was given, and he was ordered small doses of brandy and milk at frequent intervals during the night. This man died in the country about nine months after the operation from secondary deposit of cancer in the lung.

Another of the patients, also under Mr. Bennett's care, is since dead, having survived the operation rather more than eight months. The case was not an encouraging one, for the malignant mass was not confined to the stomach -in fact, did not originate there, but spread from the umbilicus, where there was a mass of hard cancer, which passed inwards, 